\title{
Bezoar-induced Small Bowel Obstruction
}

\author{
Se Heon Oh, Hwan Namgung, Mi Hyun Park ${ }^{1}$, Dong-Guk Park \\ Departments of Surgery and ${ }^{1}$ Radiology, Dankook University College of Medicine, Cheonan, Korea
}

Purpose: The aim of this study was to observe the clinical features of a bezoar-induced small bowel obstruction and to investigate the role of abdominal computed tomography $(\mathrm{CT})$ in establishing the diagnosis.

Methods: We retrospectively reviewed 20 cases of bezoar-induced small bowel obstruction in our hospital from 1996 to 2010.

Results: Thirteen patients (65\%) had a history of abdominal surgery. Nine patients (45\%) were diagnosed with a bezoar before surgery, seven patients were diagnosed by using abdominal CT, and two patients were diagnosed with a small bowel series. Abdominal CT was performed in 15 patients, and the diagnostic accuracy was $47 \%$ (7/15). Surgery revealed ten bezoars in the jejunum and 11 in the ileum. Two patients had bezoars found concurrently in the stomach. Spontaneous removal took place in two patients. An enterotomy and bezoar extraction was performed in 15 patients. Fragmentation and milking, a small bowel resection, and a Meckel's diverticulectomy were performed in one patient each. Early operative treatment was possible $(\mathrm{P}=0.036)$ once the bezoar had been diagnosed by using abdominal CT. There tended to be fewer postoperative complications in patients who were diagnosed with a bezoar by using abdominal CT, but the result was not statistically significant $(\mathrm{P}=0.712)$.

Conclusion: A preoperative diagnosis of bezoar-induced small bowel obstruction by using clinical features was difficult. Increased use of abdominal CT led to a more accurate diagnosis and to earlier surgery for bezoar-induced small bowel obstructions, thereby reducing the rate of complications.

Keywords: Bezoars; Small intestine, Intestinal obstruction; Computed tomography

\section{INTRODUCTION}

Bezoars are masses formed from mixed substances in the gastrointestinal system, and they contribute to four percent of small bowel obstructions. Bezoar-induced small bowel obstruction does not show significant clinical difference from bowel obstruction induced by other causes. Therefore, most of the cases are diagnosed postoperatively. When the diagnosis is delayed, the prevalence of fatal complications can be increased. In recent years, the use of com-

Received: October 7, 2011 Revised: November 10, 2011

Accepted: November 20, 2011

Correspondence to: Hwan Namgung, M.D.

Department of Surgery, Dankook University College of Medicine,

119 Dandae-ro, Dongnam-gu, Cheonan 330-997, Korea

Tel: +82-41-550-3929, Fax: +82-41-556-3878

E-mail: gsnamgung@dankook.ac.kr

(C) 2012 The Korean Society of Coloproctology

This is an open-access article distributed under the terms of the Creative Commons Attribution NonCommercial License (http://creativecommons.org/licenses/by-nc/3.0) which permits unrestricted noncommercial use, distribution, and reproduction in any medium, provided the original work is properly cited. puted tomography (CT) has been introduced to diagnose bowel obstruction and has increased the rate of preoperative diagnosis of bezoar-induced small bowel obstruction. Hence, the authors of this study aim to investigate the efficacy and the roles of abdominal CT in the diagnosis of bezoar-induced small bowel obstruction through clinical observations of 20 cases of bezoar-induced small bowel obstruction.

\section{METHODS}

The subjects were 20 patients who were diagnosed with bezoarinduced small bowel obstruction between March 1996 and September 2010. A retrospective study was performed considering the age, sex distribution, co-morbidity and past history of abdominal surgeries, clinical symptoms and physical examination, radiological findings, and postoperative complications through a review of the medical records. The statistical analysis was performed using SPSS ver. 12.0 (SPSS Inc., Chicago, IL, USA). The periods from the diagnosis of a bezoar to surgical intervention after an abdominal CT scan were compared using the Mann-Whitney test, and Fish- 
er's exact test was used for comparisons of complications. A statistically significant $\mathrm{P}$-value was set at less than 0.05 .

\section{RESULTS}

\section{Frequency}

The gender ratio of the patients was 3:1 (male:female), 15 male patients and 5 female patients, with an average age of 56 years (mean, 32 to 85 years). According to the monthly prevalence of the disease, three cases occurred in October, 3 cases in November, 4 cases in December and 4 cases in January, showing a 70\% incidence rate during autumn and winter (Fig. 1).

\section{History}

Thirteen patients (65\%) had past histories of abdominal surgeries: 4 cases of a vagotomy and pyroplasty, 2 cases of a subtotal gastrectomy, and 1 case each of a gastrojejunostomy, a splenectomy, a choledocojejunostomy, a laparoscopic cholecystectomy and appendectomy, and an ovarian cystectomy. The names of operations were not identified in a case of gastric perforation of a peptic ulcer and a case of bowel perforation caused by a traffic accident.

\section{Clinical signs and symptoms}

Abdominal pain was the most common symptom (90\%), followed by vomiting $(70 \%)$ and nausea (40\%). Seven patients (35\%) were admitted to the hospital within 24 hours after the onset of the symptoms, 12 patients (60\%) took one day to one week, and 1 patient (5\%) took longer than two weeks.

\section{Diagnosis}

The bezoar was identified preoperatively in 9 cases (45\%), and 7 cases among them were diagnosed by using abdominal CT and 2 cases were diagnosed by using a small bowel series. Abdominal $\mathrm{CT}$ was performed in 15 patients, and a bezoar was identified in 7 cases, showing a $47 \%$ diagnostic rate. The other 11 patients were diagnosed during surgery (Fig. 2).

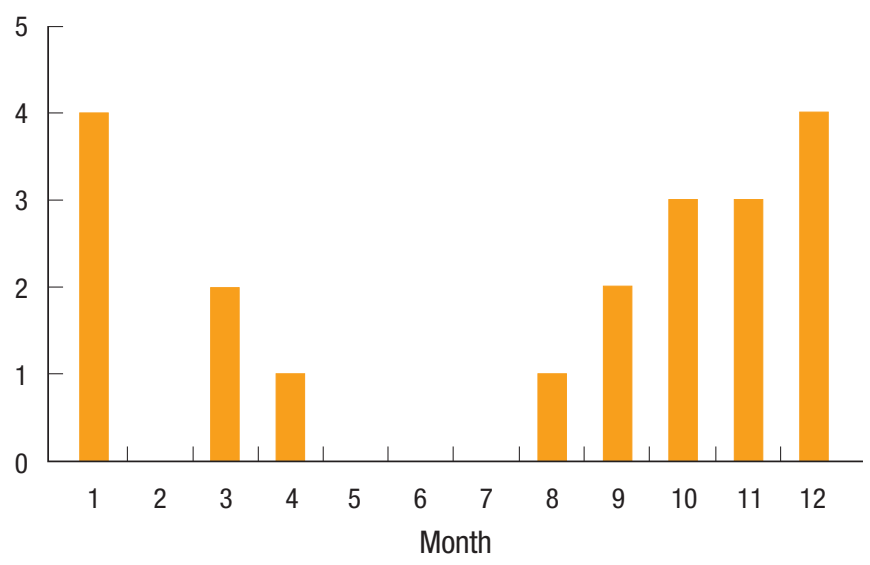

Fig. 1. Monthly incidence of bezoar-induced small bowel obstruction.
The location and the characteristics of bezoar

Eighteen patients who underwent surgery had 21 bezoars located in the small bowel, 10 of which were in the jejunum and 11 were in the ileum. The bezoars in the jejunum were distributed evenly while the bezoars in the ileum were all located within $100 \mathrm{~cm}$ from the ileocecal valve. Sixteen cases (89\%) involved solitary bezoars, and 2 cases involved multiple bezoars. A gastric bezoar was found in each of the 2 cases of multiple bezoars. All 21 cases involved phytobezoars, and the average size of the bezoars was $5.5 \mathrm{~cm}$ (mean, 3 to $10 \mathrm{~cm}$ ) (Table 1 ).

\section{Treatments}

Among 20 patients, 18 patients had surgical interventions, and the other 2 cases with smaller-sized bezoars $(3 \mathrm{~cm}, 4 \mathrm{~cm})$ located in the distal ileum were resolved by natural excretion. A jejunotomy and extraction was performed for all 7 cases of jejunal bezoars, and one of them was done by laparoscopy. A case involving an ileal bezoar was treated by using an ileotomy after the bezoar in the jejunum had been pushed to the ileum. Among 11 cases of ileal bezoars, eight cases were treated by using an ileotomy and extraction, case in which a bezoar was identified in Meckel's diverticulum was treated by using Meckel's diverticulectomy, one case was treated by fragmentation and milking, and the last was treated using an ileal resection and anastomosis. One case of a gastric bezoar identified preoperatively was treated with endoscopy, and one case of a gastric bezoar identified during surgery was treated by using a gastrotomy.

\section{Treatment outcome comparisons according to the diagnostic methods}

The patients were classified into three groups depending on the

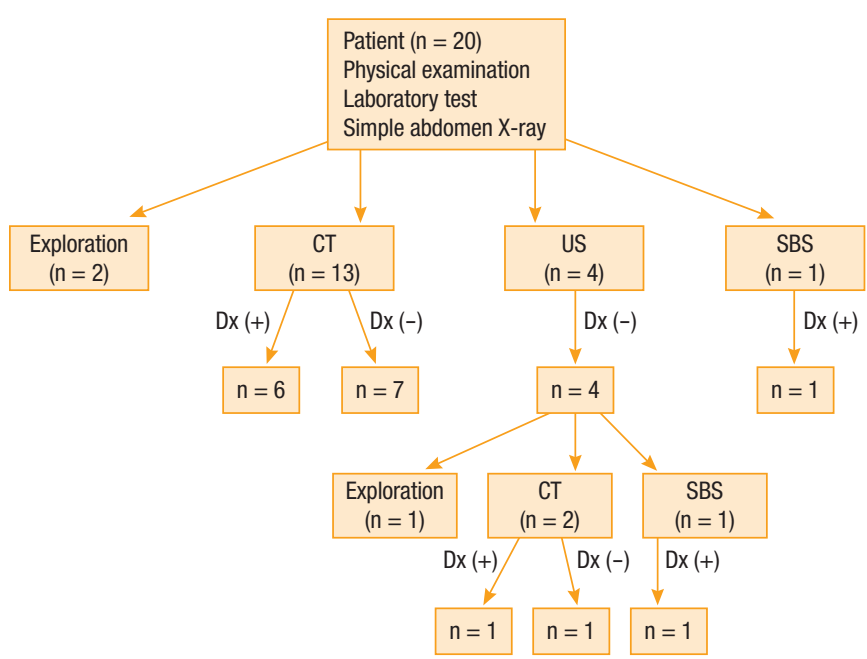

Fig. 2. Diagnostic algorithm of bezoar-induced small bowel obstruction. CT, computed tomography; US, ultrasound; SBS, small bowel series; Dx, diagnosis. 
Volume 28, Number 2, 2012

implementation of $\mathrm{CT}$ and on preoperative bezoar diagnosis to compare the periods of hospital stay and postoperative complications: the group without a CT scan (group I), the group which underwent a CT scan without a bezoar being identified (group II), and the group diagnosed with a bezoar by using the CT scan (group III). There were no significant differences in the periods of hospital stay between the groups, but the group diagnosed with a bezoar by using the CT scan had earlier surgery compared to the group that underwent a CT scan without finding a bezoar (0.6 days vs. 4.9 days, $\mathrm{P}=0.036$ ).

Among 18 patients who had surgical interventions, ten postoperative complications were identified in 6 patients: five wound infections, two postoperative bowel obstructions, 2 cases of hematochezia of unknown origin, and 1 case of bleeding from a duodenal ulcer, which required a blood transfusion. The postoperative complications in the group diagnosed with a bezoar by using the CT scan were lower than they were in the other groups, but the difference was not statistically significant ( $40 \%$ vs. $37.5 \%$ vs.

Table 1. Site of bezoars in bezoar-induced small bowel obstruction

\begin{tabular}{lc}
\hline Site of bezoars & Case $(\mathrm{n}=18)$ \\
\hline Jejunum (single) & 6 \\
lleum (single) & 10 \\
Jejunum (3) + stomach (6) & 1 \\
Jejunum (1) + ileum (1) + stomach (3) & 1 \\
\hline
\end{tabular}

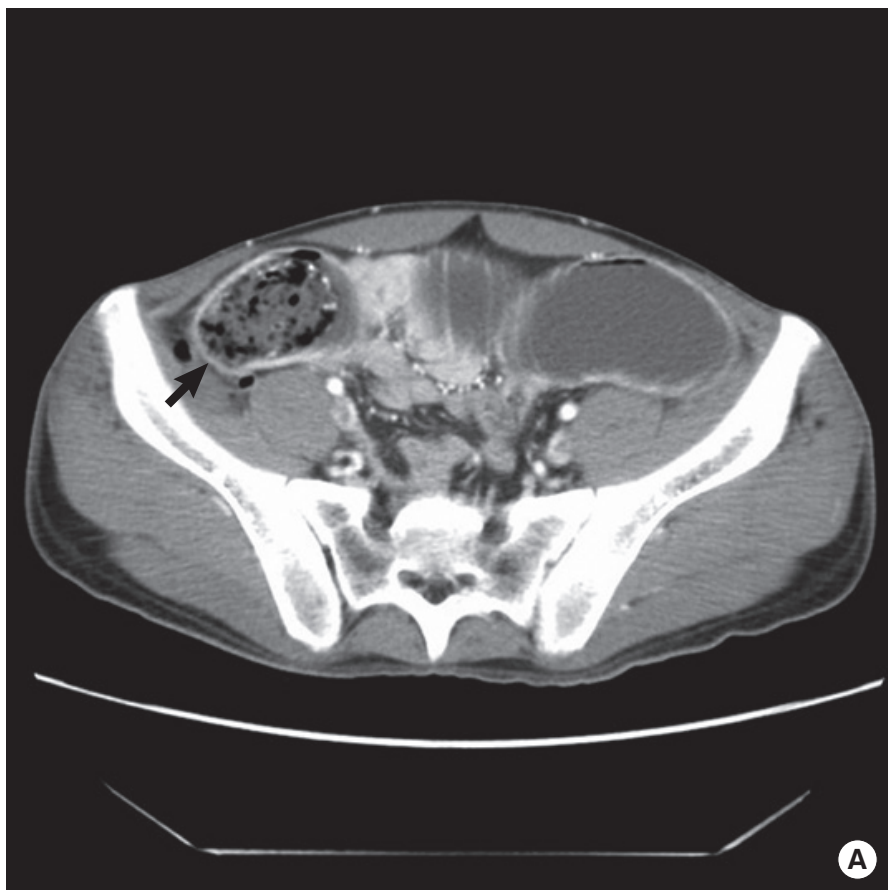

20\%, P = 0.712) (Table 2).

\section{DISCUSSION}

Four percent of small bowel obstructions are caused by bezoars, which can be formed due to large fiber intake, inadequate mastication, hasty swallowing, reduced gastric motility and pyloric dysfunction. Therefore, bezoars are prevalent among people with delayed gastric emptying such as after a gastrectomy or a vagotomy, or as a result of diabetic autonomic neuropathy and hypothyroidism. In this study, 13 cases (65\%) had a history of abdominal surgery, and co-morbidities existed, including 6 cases of ulcers (30\%), 2 cases of early stage of gastric cancer (10\%), and 2 cases of diabetes mellitus (10\%). Bezoars are identified mainly in the stomach and the small bowel. Although gastric bezoars are more frequent, bowel obstructions are usually caused by bezoars in the small bowel [1] Most bezoars in the small bowel are found 50 to $70 \mathrm{~cm}$ above the

Table 2. Hospital days and complications according to diagnostic modality and preoperative diagnosis

\begin{tabular}{lcccc}
\hline & $\begin{array}{c}\text { Group I } \\
(\mathrm{n}=5)\end{array}$ & $\begin{array}{c}\text { Group II } \\
(\mathrm{n}=8)\end{array}$ & $\begin{array}{c}\text { Group III } \\
(\mathrm{n}=5)\end{array}$ & P-value \\
\hline Hospital day & 23.2 & 18.0 & 20.9 & \\
CT-operation (day) & & 4.9 & 0.6 & 0.036 \\
Complication, case (\%) & $2(40.0)$ & $3(37.5)$ & $1(20.0)$ & 0.712 \\
\hline
\end{tabular}

CT, computed tomography.

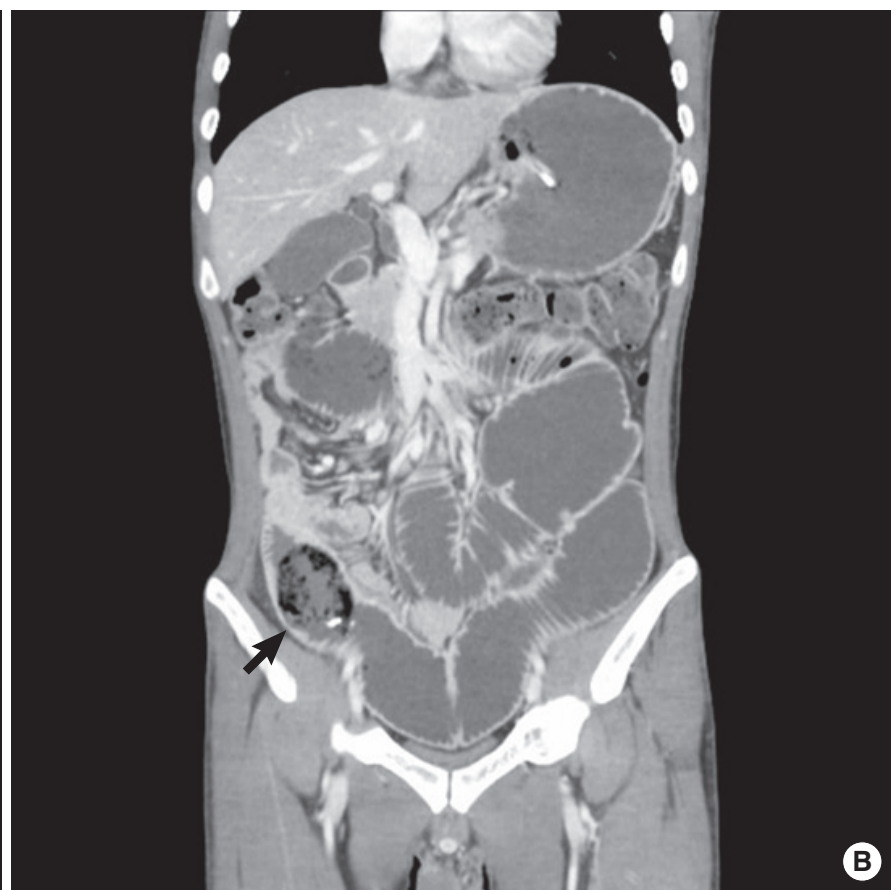

Fig. 3. Abdominal computed tomography scan shows a well-defined mass with mottled gas pattern within the transitional zone of the small bowel: (A) axial view and (B) coronal view. 
ileocecal valve because it is narrow, the intestinal motility is slower, and a large amount of water absorption hardens the bezoar, resulting in its losing its motility [2-4]. This study included 10 cases of ileal bezoars (55.7\%), 7 cases of jejunal bezoars (38.8\%) and 1 case of an ileal and jenunal multiple bezoar. A gastric bezoar was also identified in each of 2 cases of multiple bezoars (11\%); thus, checking for gastric bezoars, as well as multiple bezoars in the small bowel before or during surgery is necessary in order to prevent residual bezoars and the recurrence of small bowel obstruction after removal of the gastrointestinal bezoar [5].

The symptoms of bezoars can differ according to size, location and level of obstruction. However, gastric bezoars usually present with frequent vomiting, upper abdominal pain and abdominal distension whereas bezoars in the small bowel usually present with abdominal pain, abdominal distension, and nausea and vomiting, which are the symptoms of bowel obstruction. DeBakey and Ochsner [6] reported that upper abdominal pain was the most common symptom $(70.2 \%)$ and that nausea, vomiting, a palpable mass, and weight loss could also be seen. Also, in this study, abdominal pain was the most common symptom (90.0\%), followed by vomiting (70.0\%) and nausea (40.0\%). Differentiating bowel obstruction caused by adhesion from bowel obstruction caused by a bezoar is not easy in the patients who had previous abdominal surgery and present with symptoms of bowel obstruction. In this study, only $45 \%$ of the patients were diagnosed with bezoar-induced small bowel obstruction preoperatively. Moreover, surgical interventions can be delayed and complications will be increased if bezoar-induced small bowel obstruction is misdiagnosed as bowel obstruction caused by adhesion, which may be treated with conservative managements [7]. Therefore, the importance of early diagnosis through radiological investigation is emphasized.

There is a study reporting the diagnosis of bezoar-induced small bowel obstruction with abdominal X-ray [7], but there wasn't any evidence suggesting a bezoar other than signs of small bowel obstruction in this study. The diagnostic rate of abdominal ultrasonography is reported to be 88 to $93 \%$, but it has a drawback of being operator-dependent and a bezoar can be concealed due to the accumulated gas in the bowels affecting the ultrasonic sound $[5,8]$. In this study, abdominal ultrasonography was performed for 4 patients, but bezoars were not identified in any of the cases. A small bowel series has an excellent accuracy in differentiating bowel obstruction caused by adhesion from bowel obstruction caused by a bezoar, but it has limited applicability. It can be attempted in cases of partial bowel obstruction, but the test is not feasible in cases of complete bowel obstruction or suspected ischemia due to the high risk of bowel perforation; additionally, the contrast used for the test can cause complications in the planned operation [7]. Abdominal CT is quick and operator-independent and has superior ability to confirm the cause of the bowel obstruction, the location and the level of the obstruction, and the existence of multiple bezoars, as well as to find complications such as bowel ischemia and bowel perforation $[7,9]$. The characteristics of be- zoar-induced bowel obstruction on a CT scan can be described as a mass with mottled gas in the transition point (Fig. 3) [5], and emergency surgery should be considered if there is any evidence of submucosal edema around the bowel where the bezoar is located, pressure necrosis, perforation or strangulation $[5,9]$. The diagnostic rate of abdominal CT in diagnosing the cause of bowel obstruction is reported to be 73 to $95 \%$ [7], and its diagnostic accuracy for bezoar-induced bowel obstruction is about 65 to $100 \%$ [5]. In this study, the diagnostic rate of bezoar-induced small bowel obstruction was only $47 \%$ (7/15), which was less than in other studies. This is thought to be due to feces in the small bowel looking similar to the bezoar on CT images. The features of small bowel feces that differentiate it from a bezoar are its being present in longer segments and its being less likely to form a mass or encapsulating wall. Also, it is not located at the transition points [10]. The use of a soft-tissue window setting on the analysis of CT images can help to differentiate small bowel feces from a bezoar $[5,10,11]$. The CT images were retrospectively reviewed again by radiologists during this study, and bezoar-induced bowel obstruction was identified in all cases. Therefore, providing the radiologist with the clinician's suspicion and with sufficient information based on the patient's history and clinical symptoms, which will enable a more accurate analysis of CT images, is important.

Surgical interventions are usually required for a bezoar-induced small bowel obstruction [9]. Fragmentation and milking can be primarily attempted, and an enterotomy and bezoar extraction is performed when is the former fails [12]. Small bowel resection and anastomosis should be performed if bowel ischemia and necrosis are suspected [13]. In this study, the bowel obstruction of one patient was initially thought to be caused by adhesion; hence, it was treated with conservative management, observing the improvement. The patient eventually underwent surgery and showed bowel necrosis, leading to an ileal resection and anastomosis. Laparoscopy has recently been attempted, limitedly, to treat bowel obstructions induced by bezoars [14]. In this study, a jejunotomy and bezoar extraction was performed by laparoscopy in one patient.

In order to examine the dependence of the difference in the treatment outcomes on the implementation of abdominal CT and on the diagnosis of a bezoar, in this study, we compared the periods of hospital stay, early surgical interventions, and postoperative complications, after classifying the subjects into three groups: the group without a CT scan (group I), the group with a CT scan without a bezoar being identified (group II), and the group diagnosed with a bezoar by using the CT scan (group III). There was no difference in the periods of a hospital stay among the groups, but early surgical interventions were facilitated by the diagnosis of bezoar with abdominal CT. The cases in which the patient was admitted for a longer time before taking a CT scan and the cases in which the patient was admitted for a longer period of time in the hospital due to complications (52 days) were included in group III, which may be the reason for the similar lengths of hospital stay. The frequency of postoperative complications secondary to bowel isch- 
emia and bowel necrosis is reported to be higher when the diagnosis of bezoar-induced bowel obstruction is delayed [15]. The patients diagnosed with a bezoar by using the abdominal CT scan, who were able to receive early surgical interventions in this study, had fewer complications compared to the other groups, but the differences between the groups were not statistically significant. However, verifying the statistical significance in this study was difficult because of the small size of the sample. Therefore, further study with a larger sample will be required for verification.

In conclusion, diagnosing bezoar-induced small bowel obstruction with clinical symptoms and signs was difficult. Increased use of abdominal CT should lead to more accurate diagnosis and early surgery for bezoar-induced small bowel obstruction, thereby reducing the rate of complications. However, sufficient clinical information from clinicians and careful reading from radiologists is vital for improving the diagnostic rate of abdominal CT scans for bezoarinduced small bowel obstruction.

\section{CONFLICT OF INTEREST}

No potential conflict of interest relevant to this article was reported.

\section{REFERENCES}

1. Hong SK, Lim TJ, Park YK. A clinical study of bezoars (108 cases). Keimyung Med J 1986;5:68-73.

2. Jeong YH, Moon TI, Rhee JK, Chae KM. Clinical analysis of 17 cases of bezoar. Korean J Gastroenterol 1989;21:572-6.

3. Lee SG, Lee HY, Park KJ, Kim SH, Kim MC, Choi HJ, et al. The clinical analysis of 25 cases of bezoars. J Korean Surg Soc 2005; 68:407-13.

4. Park JS, Lee JI, Jeong JH, Lee JH, Moon HJ, Park JK, et al. The clinical analysis of 12 cases of bezoars. J Korean Surg Soc 2009; 77:177-83.
5. Bae KS, Jeon KN, Ryeom HK. Bezoar associated with small bowel obstruction: comparison of CT and US. J Korean Radiol Soc 2003; 48:53-8.

6. DeBakey M, Ochsner A. Bezoars and concretions. Surgery 1938; 4:934-64.

7. Ho TW, Koh DC. Small-bowel obstruction secondary to bezoar impaction: a diagnostic dilemma. World J Surg 2007;31:1072-8.

8. Ripolles T, Garcia-Aguayo J, Martinez MJ, Gil P. Gastrointestinal bezoars: sonographic and CT characteristics. AJR Am J Roentgenol 2001;177:65-9.

9. Kim JH, Ha HK, Sohn MJ, Kim AY, Kim TK, Kim PN, et al. CT findings of phytobezoar associated with small bowel obstruction. Eur Radiol 2003;13:299-304.

10. Zissin R, Osadchy A, Gutman V, Rathaus V, Shapiro-Feinberg M, Gayer G. CT findings in patients with small bowel obstruction due to phytobezoar. Emerg Radiol 2004;10:197-200.

11. Delabrousse E, Lubrano J, Sailley N, Aubry S, Mantion GA, Kastler BA. Small-bowel bezoar versus small-bowel feces: CT evaluation. AJR Am J Roentgenol 2008;191:1465-8.

12. Dirican A, Unal B, Tatli F, Sofotli I, Ozgor D, Piskin T, et al. Surgical treatment of phytobezoars causes acute small intestinal obstruction. Bratisl Lek Listy 2009;110:158-61.

13. Bedioui H, Daghfous A, Ayadi M, Noomen R, Chebbi F, Rebai W, et al. A report of 15 cases of small-bowel obstruction secondary to phytobezoars: predisposing factors and diagnostic difficulties. Gastroenterol Clin Biol 2008;32:596-600.

14. Yau KK, Siu WT, Law BK, Cheung HY, Ha JP, Li MK. Laparoscopic approach compared with conventional open approach for bezoar-induced small-bowel obstruction. Arch Surg 2005;140: 972-5.

15. Escamilla C, Robles-Campos R, Parrilla-Paricio P, Lujan-Mompean J, Liron-Ruiz R, Torralba-Martinez JA. Intestinal obstruction and bezoars. J Am Coll Surg 1994;179:285-8. 